\title{
Vascular Ligation in Laparoscopic Radical Nephrectomy: Comparison of the Endo GIA Stapler and Hem-o-lok Polymer Clips
}

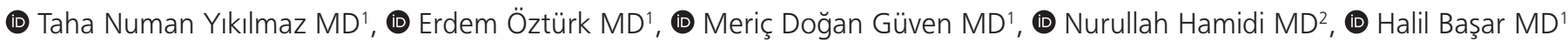 \\ 'Dr. Abdurrahman Yurtaslan Ankara Oncology Training and Research Hospital, Clinic of Urology, Ankara, Turkey \\ ${ }^{2}$ Atatürk Training and Research Hospital, Clinic of Urology, Ankara, Turkey
}

\begin{abstract}
Objective: Various techniques for vascular ligation are used during laparoscopic surgery. We aimed to compare the Endo GIA stapler and Hem-o-lok polymer clips in laparoscopic radical nephrectomy.

Materials and Methods: The charts and costs were reviewed for all 56 patients who underwent laparoscopic radical nephrectomy at our institution from May 2014 to October 2017. Patients' demographic characteristics, tumor size, blood loss, complications, operative time, and length of hospital stay were evaluated retrospectively. The obtained data were compared statistically.

Results: Forty-six patients eligible for the study were divided into 2 groups according to the use of vascular staples or polymer clips. Amount of blood loss, postoperative drainage volume, and operative time were lower in cases using vascular stapler, while polymer clips were advantageous in terms of cost.

Conclusion: Both techniques have advantages in terms of vascular ligation in laparoscopic radical nephrectomy. These techniques may be chosen based on surgeon preference or patient compatibility; however, it is more appropriate to use the material with which the surgeon is experienced.

Keywords: Nephrectomy, laparoscopic, stapler, clips, ligation
\end{abstract}

\section{Introduction}

Renal tumors are the third most common cancer of the urogenital system and are associated with the highest mortality. They are resistant to chemotherapy and radiotherapy, and the standard treatment method is surgery. Surgery can be open, laparoscopic, or robotic. Laparoscopic nephrectomy was first described by Clayman et al. (1) in 1990 and is currently practiced safely and effectively in many centers. Laparoscopic surgery, which is recommended for stage 1 and 2 renal tumors in current guidelines, can also be performed in cases of larger and more advanced stage tumors (1). These operations can be performed using retroperitoneal, transperitoneal, and hand-assisted methods, with no differences in terms of their effectiveness or reliability $(2,3)$. Compared to open nephrectomy, laparoscopic surgery has the advantages of being less painful and requiring shorter hospitalization and recovery times, while the high cost, long learning curve, and difficulty of vascular control are considered its disadvantages (4). Starting with the use of metal clips in 1993, various vascular control techniques and materials have been developed to date (2).

In this study, we evaluated differences in vascular control achieved with the Endo GIA stapler and Hem-o-lok polymer clips in laparoscopic nephrectomies performed in our center. 


\section{Materials and Methods}

Fifty-six patients who underwent laparoscopic radical nephrectomy (LRN) due to renal tumors between May 2014 and October 2017 were included in the study. The patients' demographic characteristics, tumor size, volume of blood loss, complications, operative time, and length of hospital stay were examined retrospectively. The patients were divided into 2 groups based on the use of Endo GIA stapler (Covidien Endo GIA Universal Articulating Loading Unit $30 \mathrm{~mm} 2.5$ $\mathrm{mm}$ ) or Hem-o-lok polymer clips (Weck Hem-o-lok Polymer Ligating Clips) for renal pedicle control. Patients in whom both materials were used were excluded from the study. All patients underwent LRN via transperitoneal approach. In patients for whom an Endo GIA stapler was used, the renal artery and vein were ligated without separate dissection. The inferior aspect of the renal vein was dissected until the psoas muscle became visible, and the superior aspect was freed by blunt dissection to enable the upper jaw of the vascular stapler to be positioned in Morison's pouch. The pulse of the renal vein was sensed, and the tip of the Endo GIA stapler was placed so as to rest against the psoas muscle. The stapler was activated to perform ligation. In patients on whom Hem-o-lok clips were used, the artery and vein were dissected separately and 6 clips were placed, 1 distally and 2 proximally on each vessel. After achieving renal pedicle control, the nephrectomy procedure was completed by freeing the kidney from surrounding tissues. Operative time, amount of blood loss, and total drainage volume collected during and after the operation were recorded for each patient. The data were compared between the groups.

Ethical approval was not sought for this retrospective study. Written informed consent was obtained from the patients.

\section{Statistical Analysis}

Statistical analyses were conducted using SPSS version 16.0 (SPSS Inc. Chicago, IL, USA), and the Mann-Whitney U test was used for intergroup analysis. A p value below 0.05 was regarded as significant.

\section{Results}

Of the 56 patients included in the study, 10 were excluded due to missing data, the use of both ligation methods together, or inability to complete the surgery laparoscopically. Therefore, a total of 46 patients were included in our analysis. The mean age of the patients was $69.8 \pm 2.1$ years (32-81 years) and the male/ female ratio was $26 / 20$. The patients' demographic and tumor characteristics are shown in detail in Table 1. Ligation was done using an Endo GIA stapler in group $1(n=20)$ and with Hemo-lok polymer clips in group $2(n=26)$. Extent of bleeding was compared between the groups by evaluating change from preto postoperative hemoglobin $(\mathrm{Hb})$ values and calculating intraand postoperative drainage volumes. The mean decrease in $\mathrm{Hb}$ was $1.1 \pm 0.3 \mathrm{~g} / \mathrm{dL}(8 \%)$ in group 1 and $2.7 \pm 0.9 \mathrm{~g} / \mathrm{dL}(18.8 \%)$ in group 2. The mean intraoperative drainage volume was similar in both groups ( $316 \pm 45 \mathrm{cc}$ and $354 \pm 48 \mathrm{cc}$, respectively). Postoperative drainage volume indicated significantly less bleeding in group 1 (183 \pm 27 cc and $273 \pm 29$ cc, respectively; $\mathrm{p}<0.02)$. Length of hospital stay was similar in both patient groups ( $4.1 \pm 0.4$ days and $4.4 \pm 0.5$ days, respectively). In 1 patient for whom an Endo GIA stapler was used, intraabdominal

\begin{tabular}{|l|l|l|l|}
\hline $\begin{array}{l}\text { Table 1. Demographic characteristics and comparison of patient } \\
\text { groups who underwent laparoscopic radical nephrectomy with } \\
\text { Endo GIA stapler (group 1) and Hem-o-lok clips (group 2) }\end{array}$ \\
\hline & $\begin{array}{l}\text { Group 1 } \\
(\mathbf{n}=20)\end{array}$ & $\begin{array}{l}\text { Group 2 } \\
(\mathbf{n}=26)\end{array}$ & p \\
\hline Age (mean, years) & 67.2 & 76.2 & - \\
\hline Gender (M/F) & $9 / 11$ & $17 / 9$ & - \\
\hline Tumor size (mean, mm) & 34 & 47 & - \\
\hline Hemoglobin level & \multicolumn{3}{|l|}{} \\
\hline $\begin{array}{l}\text { Preop hemoglobin } \\
\text { Postop hemoglobin } \\
\text { Change (\%) }\end{array}$ & $\begin{array}{l}13.3 \\
8.2\end{array}$ & $\begin{array}{l}14.3 \\
18.6\end{array}$ & $<0.03$ \\
\hline Operative time (mean, minutes) & $110 \pm 10$ & $178 \pm 15$ & $<0.05$ \\
\hline $\begin{array}{l}\text { Intraoperative drainage volume } \\
\text { (mean, mL) }\end{array}$ & $316 \pm 45$ & $354 \pm 48$ & - \\
\hline $\begin{array}{l}\text { Postoperative drainage volume } \\
\text { (mean, mL) }\end{array}$ & $183 \pm 27$ & $273 \pm 29$ & $<0.02$ \\
\hline Hospital stay (mean, days) & $4.1 \pm 0.4$ & $4.4 \pm 0.5$ & - \\
\hline Cost (TL) & 332 & 126 & - \\
\hline M: Male, F: Female & & & \\
\hline
\end{tabular}

hemorrhage was suspected due to sudden development of hemodynamic instability at postoperative 6 hours. The patient underwent emergency laparotomy during which bleeding from the lumbar vein was detected and repaired. The patient was discharged with no complications on postoperative day 6 .

When the groups were compared in terms of operative time, the Endo GIA group had shorter mean operative time $(110 \pm 10 \mathrm{~min}$ vs $178 \pm 15$ min; $p<0.05)$. In terms of the cost of these materials, the price specified in the Turkish Circular on Healthcare Practices for the Endo GIA stapler used in our center (Covidien Endo GIA Universal Articulating Loading Unit $30 \mathrm{~mm} 2.5 \mathrm{~mm}$ ) is $332 \mathrm{TL}$, while that of a single Hem-o-lok clips (Weck Hem-o-lok Polymer Ligating Clips) is $21 \mathrm{TL}$. Each pack of Hem-o-lok clips contains 6 clips. The mean cost per patient was calculated as 126 TL for group 2. Therefore, the cost was higher for patients on whom the Endo GIA was used. Statistical analysis showed that the use of Hem-o-lok clips was more advantageous in terms of cost, while the amount of blood loss, postoperative drainage volume, and operative time were lower among patients for whom the Endo GIA vascular stapler was used.

\section{Discussion}

LRN, one of the reflections of minimally invasive techniques in urology, has become more common than open nephrectomy in most centers. While vascular control can be achieved by suturing in open radical nephrectomy, suturing difficulties in laparoscopic surgery have led to the development of various new techniques. These include titanium clips, Hem-o-lok clips, staplers, and laparoscopic LigaSure. There are previous studies demonstrating the advantages and disadvantages of the Endo GIA stapler and Hem-o-lok clips $(5,6)$. Chan et al. (7) used the Endo GIA stapler for ligation in LRN and reported instrumentrelated complications in 10 of 565 patients. They calculated this rate as $1.7 \%$ and argued that it is more reliable than other methods (7). Apparent advantages of the Endo GIA stapler are 
that it can be angled and is easy to open; however, the twohanded operation is a drawback compared to other methods. In studies evaluating vascular length and the effectiveness of vascular control devices, Hem-o-lok clips were shown to be advantageous in terms of avoiding aortic damage in cases with short renal artery if the trocar is placed properly (5).

In addition to vascular length, there are studies showing that vascular pressure also affects the ligation method $(6,8)$. In an experimental study by Kerbl et al. (8), titanium clips were reported to be as safe as 2-0 or 0 silk sutures. Based on their calculations, staples fail in vessels with a pressure over 237 $\mathrm{mmHg}$, leading to leakage after ligation. They concluded that in patients with systolic pressure over $200 \mathrm{mmHg}$, stapling should only be utilized with the renal vein, and the placement of metal clips was more appropriate for the renal artery (8). While no such measurement was done in our study, hemorrhage was detected in one patient on whom we used a stapler, but no leakage was observed when the stapler line was examined by laparotomy. The hemorrhage was found to originate from the lumbar veins and was considered unrelated to the ligation technique due to its distance from the suture site. Meng et al. (9) used a stapler on 15 of 97 patients, of whom 1 required conversion to open surgery due to hemorrhage, while clips were used for the other 82 patients. The researchers asserted that the clip resulted in fewer complications (9).

Studies involving cost analysis have shown that polymer clips are an advantageous material because they are cheaper than vascular staplers and more reliable than titanium. In a cost study by Bernie et al. (5), the average cost per patient to use a vascular stapler was $\$ 258$, compared to a cost of $\$ 21$ to use clips. However, when the additional $\$ 1395$ cost of the clip applier was taken into account, the total cost per patient was $\$ 49$ (5). In a study conducted in Turkey in 2010, the price of an Endo GIA stapler was calculated as $1026.95 \mathrm{TL}$ and that of a single Hem-o-lok clip was calculated as 10.59-15.32 TL depending on size, with an additional cost of 70.63-102.10 TL incurred by the use of 3 clips each for the artery and vein and another for the ureter. The main drawback of Hem-o-lock clips is the cost of the $\$ 950$ appliers, which are necessary for every size clip. However, the total calculation showed that using polymer clips is advantageous in terms of cost (10). Guazzoni et al. (11) reported reducing the per patient cost by 805 euros by using Hem-o-lok clips instead of an Endo GIA stapler on patients operated after the year 2003. Therefore, as there are no differences in terms of effectiveness and complications, the general appraisal in the literature is in favor of using clips due to their low cost (5). In our cost analysis, we did not include materials used in both groups, such as trocars, endobags, and camera sleeves, in the calculation. Since 2010, prices for outpatient and inpatient health services in state health institutions are specified in the Circular on Healthcare Practices issued by the Turkish Social Security Institution. According to Circular on Healthcare Practices, the reimbursement value of the polymer clip is $21 \mathrm{TL}$, while that of the vascular stapler has been updated as $332 \mathrm{TL}$. While the polymer clip appears to be advantageous in terms of total cost per patient, the benefits of the stapler with regard to operative time and blood loss should not be overlooked. Either technique may be chosen, based on the surgeon's preference and the patient's anatomical structure. We believe that using a stapler is a fast, easy, and reliable method in cases where the pedicle can be easily exposed.

\section{Study Limitations}

Because of the small number of cases in our study, we could not perform multiple regression analysis for risk factors affecting cost. Other limitations include the absence of subgroup analysis based on tumor size and location, and the fact that the patients' preoperative blood pressure and vessel width and length were not measured.

\section{Conclusion}

Our comparison of these 2 vascular control devices has shown that vascular stapler is advantageous in all respects except cost. Nevertheless, it seems more appropriate for the surgeon to choose the method with which he or she is experienced.

\section{Ethics}

Ethics Committee Approval: Not obtained because this study is retrospective.

Informed Consent: Written informed consent was obtained from patients.

Peer-review: Externally peer-reviewed.

\section{Authorship Contributions}

Surgical and Medical Practices: T.N.Y., E.Ö., H.B., Concept: T.N.Y., E.Ö., M.D.G., Design: T.N.Y., H.B., Data Collection or Processing: M.D.G., Analysis or Interpretation: T.N.Y., E.Ö., Literature Search: T.N.Y., N.H., Writing: T.N.Y.

Conflict of Interest: No conflict of interest was declared by the authors.

Financial Disclosure: The authors declared that this study received no financial support.

\section{References}

1. Clayman RV, Kavoussi LR, Soper NJ, et al. Laparoscopic nephrectomy: initial case report. J Urol 1991;146:278-282.

2. Cabello R, García JV, Quicios C, et al. Is there a new alternative for a safer kidney artery ligation in laparoscopic donor nephrectomy? J Laparoendosc Adv Surg Tech A 2017;27:715-716.

3. Wolf JS Jr, Moon TD, Nakada SY. Hand-assisted laparoscopic neprectomy; comparison to standart laparoscopic neprectomy. J Urol 1998; 160:22-27.

4. Nadu A, Mor Y, Chen J, et al. Laparoscopic nephrectomy; initial experience in Israel with 110 cases. Isr Med Assoc J 2005;7:431-434.

5. Bernie JE, Sundaram CP, Guise Al. Laparoscopic vascular control techniques in donor nephrectomy: Effects on vessel length. JSLS 2006;10:141-144.

6. Joseph J, Leung YY, Eichel L, et al. Comparison of the Ti-knot device and Hem-o-lok clips with other devices commonly used for laparoscopic renal-artery ligation. J Endourol 2004;18:163-166.

7. Chan D, Bishoff JT, Ratner L, et al. Endovascular gastrointestinal stapler device malfunction during laparoscopic nephrectomy: early recognition and management. J Urol 2000;164:319-321.

8. Kerbl K, Chandhoke PS, Clayman RV, et al. Ligation of the pedicle during laparoscopic nephrectomy: A comparison of staples, clips, and sutures. J Laparoendosc Surg 1993;3:9-12.

9. Meng MV, Freise CE, Kang SM, et al. Techniques to optimize vascular control during laparoscopic donor nephrectomy. Urology 2003;61:93-98.

10. Başok EK, Yıldırım A, Başaran A, ve ark. Laparoskopik ve açık böbrek cerrahisinde maliyet analizi. Turk J Urol 2008;34:100-107.

11. Guazzoni G, Cestari A, Naspro R, et al. Cost containment in laparoscopic radical nephrectomy: Feasibility and advantages over open radical nephrectomy. J Endourol 2006;20:509-513. 